\title{
Mesonic and baryonic correlation functions at fine lattice spacings
}

\author{
Stefano Capitani, Michele Della Morte, Eric Endreß, Andreas Jüttner, \\ Bastian Knippschild, Hartmut Wittig* and Manuel Zambrana \\ Institut für Kernphysik, University of Mainz, Becher Weg 45, 55099 Mainz, Germany \\ E-mail: wittigakph.uni-mainz.de
}

\begin{abstract}
We report on our on-going project to compute mesonic and baryonic two- and three-point correlation functions in simulations using $N_{\mathrm{f}}=2$ flavours of $\mathrm{O}(a)$ improved Wilson quarks and the Wilson plaquette action. We present performance figures for the DD-HMC algorithm on commodity cluster hardware and discuss the issue of critical slowing down, which is particularly pronounced for the topological charge. The effectiveness of stochastic noise sources and Jacobi smearing are investigated. Our preliminary results obtained at three quark masses on $96 \times 48^{3}$ at $\beta=5.5$ imply that the lattice spacing is about $0.06 \mathrm{fm}$, while the smallest pion mass in the current runs is around $360 \mathrm{MeV}$, which corresponds to $m_{\pi} L=5.3$.
\end{abstract}

The XXVII International Symposium on Lattice Field Theory - LAT2009

July 26-31 2009

Peking University, Beijing, China

${ }^{*}$ Speaker. 


\section{Introduction}

Despite the fact that there has been enormous recent activity in simulating lattice QCD with dynamical quarks, the continuum limit is still poorly understood. There are few systematic scaling studies of hadronic quantities, and many results for phenomenologically interesting observables have been obtained at one or two values of the lattice spacing only. As far as control over cutoff effects is concerned, simulations with dynamical quarks have not yet reached the same maturity compared to the quenched approximation. As the latter is being abandoned, one runs the risk of replacing one systematic effect (quenching) by another. The need for having full control over all systematics is further highlighted by the fact that lattice results are increasingly important for providing constraints on the validity of the Standard Model.

The work presented here is part of the CLS ("Coordinated Lattice Simulations") project [1], which is aimed at generating a set of ensembles for QCD with two dynamical flavours for a variety of lattice spacings ( $a \approx 0.04,0.06,0.08 \mathrm{fm}$ ) and volumes, such that the continuum limit can be taken in a controlled manner. Non-perturbatively $\mathrm{O}(a)$ improved Wilson quarks are used to discretise the quark action. In order to tune the masses of the light quarks towards their physical values whilst keeping the numerical effort in the simulations at a manageable level, we employ the deflationaccelerated DD-HMC algorithm [2].

\section{Production runs}

The production runs which we carry out as part of the CLS effort are performed on the cluster platform "Wilson" at the University of Mainz, which is exclusively used for lattice QCD [3]. It comprises 280 compute nodes, each equipped with two AMD 2356 "Barcelona" processors, clocked at $2.3 \mathrm{GHz}$. Each core has one GByte of memory so that the cluster's total memory amounts to 2.24 TBytes. Communication between nodes is realised via an Infiniband network and switch (DDR 20+20 Gb/s full duplex). The compute nodes are placed in water-cooled server racks. Benchmarks based on typical QCD applications [4] show that the cluster's sustained performance scales up to 3.6 TFlops, depending on the local system size. Considering the procurement costs of $1.1 \mathrm{M} €$ thus implies a cost-effectiveness of about $0.30 € /$ MFlops (sustained). The ratio of the required cooling capacity per compute speed amounts to $20 \mathrm{~kW} / \mathrm{TFlops}$.

We have generated configurations at $\beta=5.5$ on lattices of size $96 \cdot 48^{3}$. Following [5] we set the coefficient of the Sheikholeslami-Wohlert term to $c_{\mathrm{sw}}=1.75150$. On our cluster platform, we ran at three values of the hopping parameter simultaneously, using 576 processor cores per job. The length of one HMC trajectory was set to $\tau=0.5$, and the block size chosen as $8^{2} \times 12^{2}$. Further information on simulation parameters and performance figures is listed in Table 1 . At each value of $\kappa$ several thousand trajectories were generated for thermalisation.

The Monte Carlo history of the average plaquette at our largest quark mass is shown in Fig. 1. For the first 3000 trajectories a small trend in the data is observed, which is attributed to insufficient thermalisation. Similar observations were made at the other quark masses, and hence we discarded the first 3000 trajectories in each run. Following the method in ref. [6], the integrated autocorrelation time for the plaquette was determined, and the resulting values are listed in Table 1. We stored configurations after every 16th trajectory on disk, which leaves us with more than 600 configurations at each quark mass. 


\begin{tabular}{ccccccccc}
\hline \hline Run & Lattice & Block size & $\kappa$ & $n_{0}, n_{1}, n_{2}$ & time/traj. & $P_{\text {acc }}$ & $N_{\text {tr }}$ & $\tau_{\text {int }}[$ plaq $]$ \\
\hline N3 & $96 \times 48^{3}$ & $8^{2} \times 12^{2}$ & 0.13640 & $4,5,16$ & $763 \mathrm{~s}$ & 0.85 & 13761 & $16(3)$ \\
N4 & & & 0.13650 & $4,5,20$ & $943 \mathrm{~s}$ & 0.87 & 13104 & $14(2)$ \\
N5 & & & 0.13660 & $4,5,24$ & $1262 \mathrm{~s}$ & 0.86 & 12419 & $16(3)$ \\
\hline \hline
\end{tabular}

Table 1: Run parameters at $\beta=5.5$. We list the number of steps used in the hierarchical integration schemes [2], the average CPU time per trajectory, acceptance rate $P_{\text {acc }}$ and the total number of trajectories, $N_{\text {tr }}$, generated in each run. The last column contains the integrated autocorrelation time of the average plaquette, obtained after discarding the first 3000 trajectories in each run.
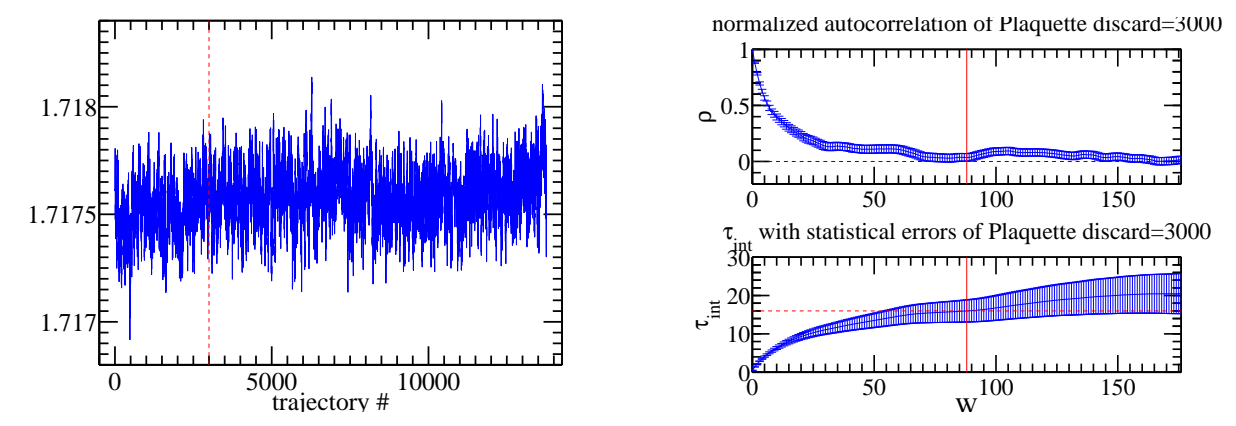

Figure 1: Left: Monte Carlo history of the average plaquette for run N3. Trajectories to the left of the vertical dashed line were discarded; Right: Autocorrelation functions of the average plaquette obtained after discarding the first 3000 trajectories.
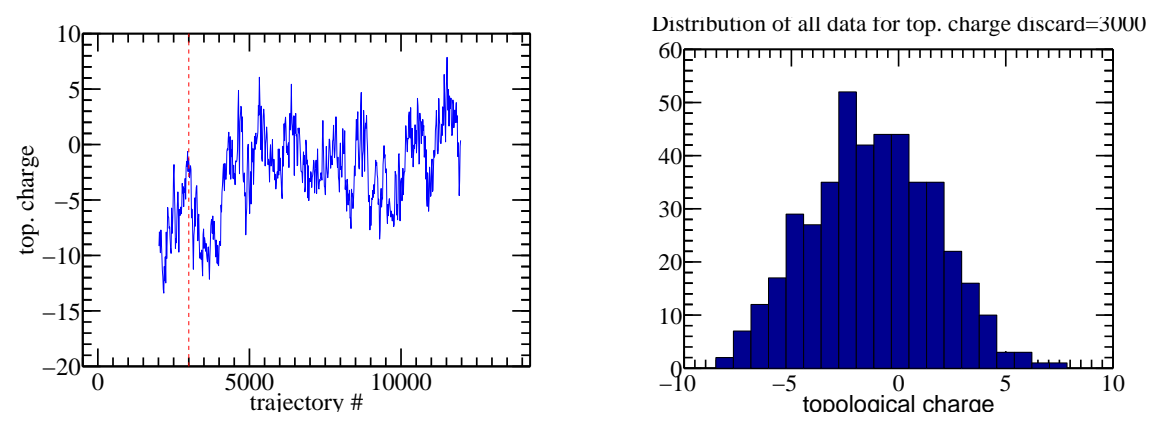

Figure 2: Left: Monte Carlo history of the topological charge for run N3; Right: Distribution of the topological charge after discarding the first 3000 trajectories.

The simulations performed as part of the CLS project revealed a severe case of critical slowing down in the topological charge, which manifests itself in a steep rise of the associated autocorrelation time as a function of the lattice spacing. In particular, it was observed [7] that at $\beta=5.7$ (which corresponds to a lattice spacing of $a \approx 0.04 \mathrm{fm}$ ), tunnelling between topological sectors is strongly suppressed. In Fig. 2 we plot the Monte Carlo history for run N3 of the topological charge, $Q=a^{4} \sum_{x} \operatorname{tr}[F(x) \tilde{F}(x)] /\left(16 \pi^{2}\right)$. With the exception of the first 3-4000 trajectories, the topological charge does fluctuate around zero at a sizeable rate and produces a distribution which is reasonably symmetric. Similar observations were made at the other values of the quark masses used in our 
simulations. Thus, unlike the situation encountered at the larger $\beta$-value of 5.7 [7] the topological charge does not appear to be stuck in a particular sector. While this may be accidental, we can take confidence that the composition of our ensembles is apparently not strongly biased. We stress that critical slowing is a general problem for lattice simulations near the continuum, which calls for a radical treatment like the one proposed in [8].

\section{Mesonic and baryonic two-point functions}

The most widely used procedure to compute quark propagators is the source method, which amounts to solving the linear system

$$
D \Phi=\eta
$$

where $D$ is the lattice Dirac operator and $\eta$ a source vector. If $\eta$ is chosen to be a point source, the resulting hadron correlators can be quite noisy, with the exception of the simplest channels such as the pion. An unambiguous identification of the asymptotic behaviour is then quite difficult. It is not only desirable to reduce the level of statistical noise but also to enhance the spectral weight of the desired state in the spectral decomposition of the correlator. In our simulations we have addressed the first problem by comparing different stochastic noise sources [9]. In particular, we have implemented the generalised "one-end-trick" [10]. In order to enhance and tune the projection properties of interpolating operators, we have implemented several variants of Jacobi smearing [11].

Let $\eta$ be a random noise vector which satisfies

$$
\left\langle\left\langle\eta_{\alpha}^{a}(x) \eta_{\beta}^{b *}(y)\right\rangle\right\rangle=\delta^{(4)}(x-y) \delta^{a b} \delta_{\alpha \beta},
$$

where double brackets denote the stochastic average. The two-point correlation function of a quark bilinear, $O(x)=(\bar{\psi} \Gamma \psi)(x)$ is given by

$$
\sum_{\vec{x}, \vec{y}}\left\langle O(x) O(y)^{\dagger}\right\rangle=-\left\langle\operatorname{Tr}\left\{S(x, y) \Gamma \gamma_{5} S(x, y)^{\dagger} \gamma_{5} \tilde{\Gamma}\right\}\right\rangle,
$$

where $\tilde{\Gamma}=\gamma_{0} \Gamma^{\dagger} \gamma_{0}$. The generalised one-end-trick amounts to choosing a spin-diagonal random source vector. More specifically, the noise source has support only on a particular spin component $\tau$ and timeslice (e.g. $y_{0}=0$ ), viz.

$$
\eta_{\sigma}^{b}(y)=\xi^{b}(\vec{y}) \delta_{0 y_{0}} \delta_{\sigma \tau}, \quad\left\langle\left\langle\xi^{b}(\vec{y}) \xi^{c *}(\vec{z})\right\rangle\right\rangle=\delta^{(3)}(\vec{y}-\vec{z}) \delta^{b c} .
$$

Solving the linear system, eq. (3.1), for spin component $\tau$ yields the solution vector $\Phi$, i.e.

$$
\Phi_{\alpha ; \tau}^{a}(x)=\left.\sum_{\vec{y}} \sum_{b} S_{\alpha \tau}^{a b}(x, y)\right|_{y_{0}=0} \xi^{b}(\vec{y}) .
$$

The correlation function is then obtained as

$$
\sum_{\vec{x}, \vec{y}}\left\langle O(x) O(y)^{\dagger}\right\rangle=-\left\langle\sum_{\vec{x}} \sum_{a, \alpha, \tau}\left\langle\left\langle\left[\left(\Gamma \gamma_{5}\right) \Phi(x)^{\dagger}\right]_{\tau ; \alpha}^{a}\left[\left(\gamma_{5} \tilde{\Gamma}\right) \Phi(x)\right]_{\alpha ; \tau}^{a}\right\rangle\right\rangle\right\rangle,
$$

For every "hit", i.e. every choice of random source one must perform four inversions, one for each spin component $\tau$. Compared with the point source, the numerical effort is reduced by a factor three per hit. 

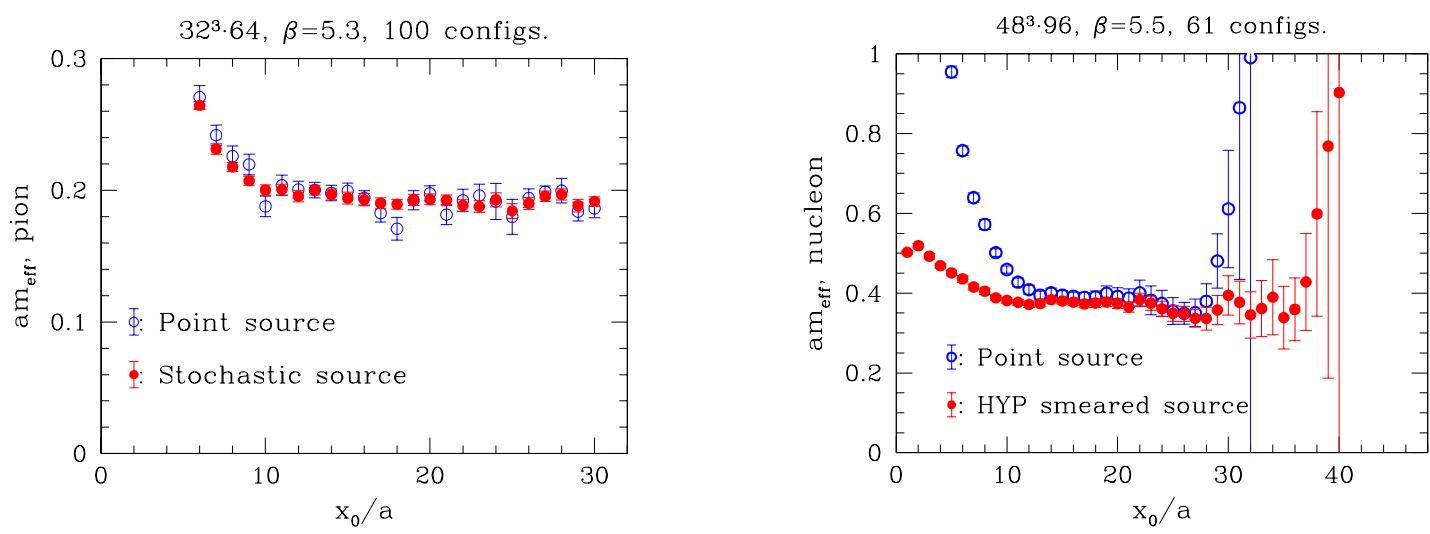

Figure 3: Left: Effective masses in the pion channel obtained using a point source and spin-diagonal $Z_{2} \otimes Z_{2}$ noise source at fixed numerical cost; Right: Effective masses for the nucleon computed on lattice N4.

In our project we have chosen $Z_{2} \otimes Z_{2}$ noise for the sources $\xi$. In Fig. 3 we compare the statistical signal for the conventional point source to the generalised one-end trick for three hits, such that the numerical cost for computing correlators for the two source types is identical. It is seen that in the pion channel random noise sources can lead to a significant enhancement of the statistical signal. Further studies showed that a similar improvement is, unfortunately, not observed in the vector channel. For baryons, we used the method of ref. [12], but without explicit low-mode averaging. Here, in order to reach a given statistical accuracy, the numerical effort was at least as large as for point sources, even after trying various dilution schemes [13], and therefore we found the method to be practically useless for the determination of baryonic ground state masses.

In order to enhance the projection onto the ground state in a given channel, particularly for baryons, we have implemented Jacobi smearing [11], supplemented by "fat" link variables. The latter were obtained either via the APE [14] or via the HYP [15] procedure. While we found much better plateaus when using smeared links of either type, HYP smearing appears to have a slight advantage. In Fig. 3 we compare effective mass plots for the nucleon, computed using point and HYP-Jacobi smeared sources. It is seen that not only the contribution of excited states is reduced but that also the plateau extends to larger timeslices if HYP-Jacobi smearing is applied, although there may be room for further improvement via better tuning of the smearing parameters.

\section{Setting the scale}

In order to convert the pion masses computed on our ensembles into physical units, we must set the scale. The mass of the $\Omega$ baryon is very well suited for this purpose, since the $\Omega$ is stable in QCD and because it contains only strange quarks in the valence sector. A long chiral extrapolation in the valence quark mass can thus be avoided. For a reliable determination of the mass of the $\Omega$, however, our simulations and analyses are not yet advanced enough. In order to obtain preliminary values for the lattice scale, we have therefore resorted to using the mass of the $K^{*}$-meson.

To this end we have followed the procedure outlined in [16]: we have determined the masses of pseudoscalar and vector mesons for degenerate and non-degenerate combinations of quarks, where 


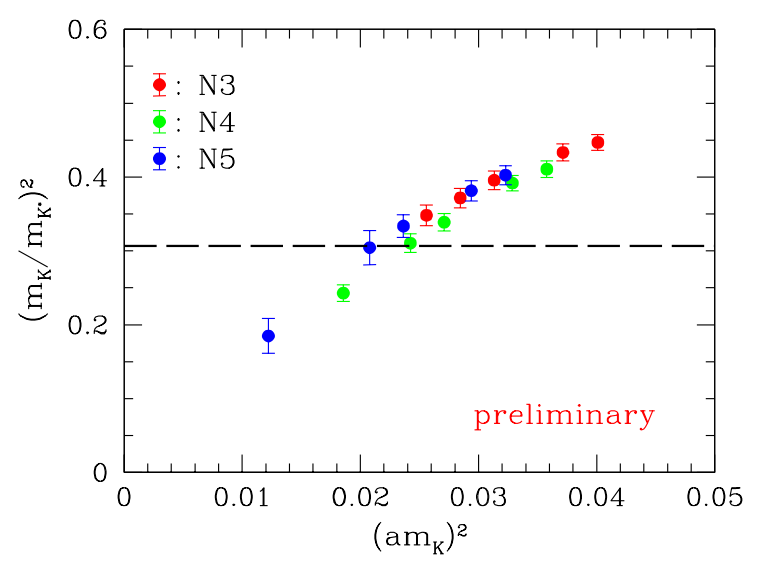

Figure 4: The ratio $\left(m_{K} / m_{K^{*}}\right)^{2}$ as a function of $\left(a m_{K}\right)^{2}$ for the three data sets. The horizontal dashed lines denotes the physical ratio $m_{K} / m_{K^{*}}=0.554$.

\begin{tabular}{ccc}
\hline Run & $m_{\pi} L$ & $m_{\pi}[\mathrm{MeV}]$ \\
\hline N3 & $7.680(15)$ & $524(13)$ \\
N4 & $6.540(18)$ & $446(11)$ \\
N5 & $5.306(23)$ & $362(9)$ \\
\hline
\end{tabular}

Table 2: Preliminary results for pion masses in physical units.

one of the masses was fixed to coincide with the sea quark mass. We denote the masses of the generic non-degenerate pseudoscalar and vector mesons by $m_{K}$ and $m_{K^{*}}$, respectively. Their values were obtained from single-exponential fits to the corresponding correlation functions, where the latter were computed using stochastic sources. The first step in the scale-setting procedure consists of interpolating the ratio $\left(m_{K} / m_{K^{*}}\right)^{2}$ as a function of $\left(a m_{K}\right)^{2}$ to the experimentally observed value of $m_{K} / m_{K^{*}}=0.554$. Fig. 4 shows the data points for the three ensembles. The intersection of the fit curves with the horizontal dashed line determines the kaon mass in lattice units, $a m_{K}$, and thus fixes the bare mass of the strange quark at a given value of the sea quark mass. In the second step one interpolates $a m_{K}$ in the sea quark mass to the reference value $m_{\pi} / m_{K}=0.85$. Obviously, this value does not correspond to the physical situation. However, as explained in [16], it serves as a perfectly well-defined reference point, which is sufficient for comparing data on different ensembles. The kaon mass at the reference point is determined as $\left.a m_{K}\right|_{\text {ref }}=0.1512(38)$. After inserting the physical (isospin-averaged) kaon mass of $495 \mathrm{MeV}$, one obtains $a=0.0603$ (15) fm. This value can then be used to convert the pion masses on the various ensembles into physical units, which yields the values listed in Table 2, where the combination $m_{\pi} L$ is shown as well.

\section{Conclusions}

Our studies have shown that large lattices at fine resolution can be simulated efficiently on commodity clusters. In spite of a sharp increase in the autocorrelation time of the topological charge observed at even smaller lattice spacings [7], the distributions for this quantity obtained in 
our runs are not pathological. We plan to compute two- and three-point correlation functions for mesonic and baryonic states in order to determine a variety of observables. So far our minimum pion mass is about $360 \mathrm{MeV}$, and $m_{\pi} L$ is kept larger than 5 . Lowering the quark mass further, in order to access pion masses of less than $300 \mathrm{MeV}$ would necessitate going to larger lattice sizes, if one wants to maintain the condition $m_{\pi} L>3$.

With the currently available algorithms, i.e. while a satisfactory solution to the problem of critical slowing down is still under investigation, it is not worth investing more effort into the generation of ensembles with smaller lattice spacings.

Acknowledgments This work was supported by Deutsche Forschungsgemeinschaft (SFB443), Gesellschaft für Schwerionenforschung, GSI, and the Research Center "Elementary Forces and Mathematical Foundations" funded by Forschungsinitiative Rheinland-Pfalz.

\section{References}

[1] https://twiki.cern.ch/twiki/bin/view/CLS/WebHome

[2] M. Lüscher, Comput. Phys. Commun. 165 (2005) 199, hep-lat/0409106; JHEP 0712 (2007) 011, arXiv:0710.5417 [hep-lat].

[3] http://wwwkph.kph.uni-mainz.de/T/644.php

[4] http://luscher.web.cern.ch/luscher/QCDpbm/index.html

[5] ALPHA Collaboration (K. Jansen and R. Sommer), Nucl. Phys. B 530 (1998) 185 [Erratum-ibid. B 643 (2002) 517], hep-lat/9803017.

[6] U. Wolff,Comput. Phys. Commun. 156 (2004) 143 [Erratum-ibid. 176 (2007) 383], hep-lat/0306017.

[7] S. Schaefer, R. Sommer and F. Virotta, Investigating the critical slowing down of QCD simulations, arXiv:0910.1465 [hep-lat].

[8] M. Lüscher, Trivializing maps, the Wilson flow and the HMC algorithm, arXiv:0907.5491 [hep-lat].

[9] E. Endreß, Computation of lattice correlation functions with reduced variance, Diploma thesis, Mainz University, 2009.

[10] UKQCD Collaboration (M. Foster and C. Michael), Phys. Rev. D 59 (1999) 074503, hep-lat/9810021; P.A. Boyle, A. Jüttner, C. Kelly and R.D. Kenway, JHEP 0808 (2008) 086, arXiv:0804.1501 [hep-lat]; C. Alexandrou and G. Koutsou, Phys. Rev. D 78 (2008) 094506, arXiv:0809.2056 [hep-lat].

[11] UKQCD Collaboration (C.R. Allton et al.), Phys. Rev. D 47 (1993) 5128, hep-lat/9303009.

[12] J. Foley, K. Jimmy Juge, A. O’Cais, M. Peardon, S.M. Ryan and J.I. Skullerud, Comput. Phys. Commun. 172 (2005) 145, hep-lat/0505023.

[13] J. Bulava, R. Edwards and C. Morningstar, Stochastic All-to-All Propagators for Baryon Correlators, arXiv:0810.1469 [hep-lat].

[14] M. Albanese et al., Phys. Lett. B 192 (1987) 163.

[15] A. Hasenfratz and F. Knechtli, Phys. Rev. D 64 (2001) 034504, hep-lat/0103029.

[16] L. Del Debbio, L. Giusti, M. Lüscher, R. Petronzio and N. Tantalo, JHEP 0702 (2007) 056, hep-lat/0610059. 